\title{
Alendronate-Induced Esophagitis: Possible Pathogenic Role of Hypersensitivity to Alendronate
}

\author{
Taio Naniwa ${ }^{1,2}$, Tomoyo Maeda ${ }^{1,2}$, Tsutomu Mizoshita ${ }^{3}$, Yoshihito Hayami ${ }^{1,2}$, \\ Maiko Watanabe ${ }^{1,2}$, Shogo Banno ${ }^{1,2}$ and Rei Ito ${ }^{1,2}$
}

\begin{abstract}
Upper gastrointestinal tract mucosal irritations, such as esophagitis, have been reported as rare adverse events due to a variety of aminobisphosphonates, including alendronate sodium, which have been widely used to treat osteoporosis. Although the pathogenesis of aminobisphosphonate-induced esophageal mucosal irritation has not been clearly understood, direct chemical esophageal irritation with prolonged local mucosal exposure to the drug with gastric contents might be the most plausible mechanism according to the previously reported literature. Here we report a young adult man with severe ulcerative esophagitis due to alendronate who demonstrated a strongly positive result on a drug lymphocyte stimulation test against alendronate. This case report provides the new concept that T-cell mediated delayed hypersensitivity to the drug may be involved in the pathogenesis of alendronate-induced esophagitis.
\end{abstract}

Key words: bisphosphonate, alendronate, esophagitis, adverse event, drug lymphocyte stimulation test

(Inter Med 47: 2083-2085, 2008)

(DOI: 10.2169/internalmedicine.47.1457)

\section{Introduction}

Alendronate, one of the bisphosphonates, is effective and widely used in the prevention and treatment of glucocorticoid-induced osteoporosis. Postmarketing surveillance of alendronate alerted that alendronate could cause chemical esophagitis, including severe ulcerations in some patients (1). Many of these cases occurred in elderly individuals or in individuals who did not follow the recommended dosing instructions (1). Here, we report a young adult case of severe ulcerative esophagitis, which developed about two days after the first intake of alendronate in spite of following recommended dosing instructions, and demonstrated a strongly positive result of drug lymphocyte stimulation test against alendronate. These findings suggested that the delayed-hypersensitivity induced by alendronate played an important role in the pathogenesis of esophagitis in this patient.

\section{Case Report}

A 30-year-old man presented to the emergency department with a 5-day history of progressive anterior chest pain, painful swallowing, and inability to swallow solids and liquids that developed two days after the initiation of treatment with weekly dosing of $35 \mathrm{mg}$ tablet of alendronate. Following the recommended dosing instructions, he took alendronate with a full glass of water and remained upright for at least 30 minutes after taking alendronate. His other medications consisted of $10 \mathrm{mg} / \mathrm{day}$ of prednisolone as maintenance therapy for Henoch-Schoenlein purpura with nephritis; $1 \mu \mathrm{g} / \mathrm{day}$ of alfacalcidol for the prevention of corticosteroid-induced osteoporosis; $50 \mathrm{mg} /$ day of fluvoxamine maleate for the depressive state. Other than the alendronate, no new medicines had been taken during the preceding six months. He had no previous history of drug allergy or bisphosphonate therapy. At presentation, he had high fever that had developed from the previous day and was unable to

\footnotetext{
${ }^{1}$ Division of Rheumatology, Nagoya City University Hospital, Nagoya, ${ }^{2}$ Department of Medical Oncology and Immunology, Nagoya City University Graduate School of Medical Sciences, Nagoya and ${ }^{3}$ Department of Gastroenterology and Metabolism, Nagoya City University Graduate School of Medical Sciences, Nagoya

Received for publication June 30, 2008; Accepted for publication August 22, 2008

Correspondence to Dr. Taio Naniwa, tnaniwa@med.nagoya-cu.ac.jp
} 
swallow his own secretions. Endoscopy showed severe, circumferential erosions and longitudinal ulcerations in the middle and distal portion of the esophagus (Fig. 1A, 1B), with a normal-appearing proximal esophagus, stomach, and duodenum. Computed tomography of the chest showed circumferential transmural thickening of the distal portion of the esophagus with inflammatory changes in the mediastinum around the thickened esophagus (Fig. 1C). Signs of hiatus hernia were not observed in endoscopic and computed tomographic findings. Laboratory studies on admission disclosed leukocytosis $\left(11,600 / \mathrm{mm}^{3}\right)$ and elevation of C-reactive protein $(8.07 \mathrm{mg} / \mathrm{dL})$. Liver and kidney functions were normal. Blood culture obtained on admission was negative. Cytomegalovirus pp65 leukocytic antigenemia, as determined using monoclonal antibody HRP-C7, was negative. As he developed esophagitis about 48 hours after first intake of alendronate with severe local and systemic inflammatory responses, including high fever, severe chest pain, and marked elevation of C-reactive protein without evidence of esophageal perforation, we suspected that he had delayed hypersensitivity reaction induced by alendronate $35 \mathrm{mg}$ tablet and checked it by drug lymphocyte stimulation test (DLST). DLST was performed by SRL, Inc. (Tokyo), which is a company that provides a comprehensive laboratory testing service in Japan. In the DLST, mitogenic activity of peripheral blood mononuclear cells was quantified by $\left[{ }^{3} \mathrm{H}\right]$ thymidine incorporation. The stimulation index (SI) is defined as the amount of the uptake of $\left[{ }^{3} \mathrm{H}\right]$ thymidine induced by the allergen divided by that induced by negative control. The cut-off value of SI for positivity provided by SRL, Inc was $180 \%$. In this patient, the blood served for drug lymphocyte stimulation test (DLST) was obtained on the third hospital day. The result of DLST was strongly positive; the uptake of $\left[{ }^{3} \mathrm{H}\right]$ thymidine induced by alendronate $35 \mathrm{mg}$ tablet was 13,714 counts per minute (cpm), while that induced by negative control was $230 \mathrm{cpm}$, thus SI was 5,962\%. The patient was treated with intravenously administered $40 \mathrm{mg} /$ day of omeprazol, up to $45 \mathrm{mg} /$ day of pentazocine, $10 \mathrm{mg} /$ day of prednine, up to $100 \mathrm{mg} /$ day of diclofenac suppositories and total parenteral nutrition. Fever subsided in the second hospital day. Two weeks later, nearly all the symptoms had resolved. Prednisolone, alfacalcidol, and fluvoxamine were re-administered with no trouble. One month later, repeated endoscopy showed marked improvement of esophagitis. Thereafter he has been free of gastrointestinal symptoms.

\section{Discussion}

Injury to the esophageal mucosa caused by tablets passing through the esophagus is a rare but well-known clinical condition known as pill-esophagitis (2). Recent reports have shown that oral bisphosphonates, including alendronate, can cause pill-esophagitis (1, 3-6)-. A large review of worldwide post-marketing surveillance data for alendronate in 1996 showed that $199(0.04 \%)$ reports of esophageal adverse events were received for an estimated 475,000 patients
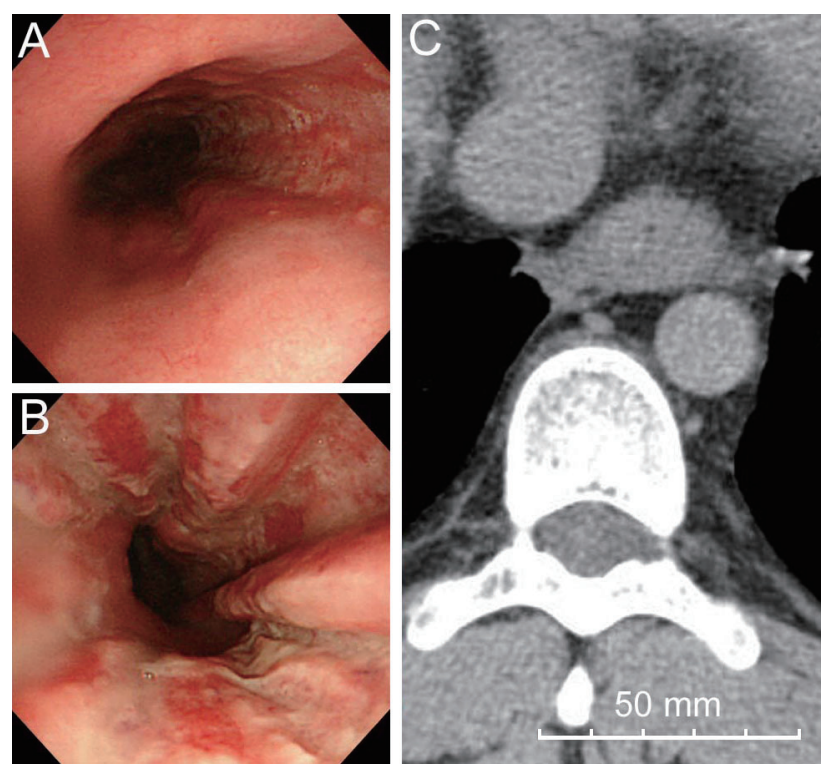

Figure 1. Endoscopic image of the esophagus (Panel A, B) and CT scan of the chest (Panel C) on admission. Panel A and $B$ show severe, circumferential erosions and longitudinal ulcerations in the middle (A) and distal (B) esophagus. Panel C shows concentric esophageal-wall thickening and circumferential high-density area around the esophagus suggestive of transmural inflammation and its extension to the tissues around the esophagus.

(spontaneous reporting to the manufacturer) (1). On the other hand, in the double-blind placebo-controlled trials for osteoporosis, the overall incidence of adverse upper gastrointestinal effects was similar in patients receiving placebo and those receiving alendronate $(3,7)$. These results indicate that the esophageal adverse effects occur in only a small number of patients.

These studies have also demonstrated that the esophageal manifestation associated with alendronate varied between cases. Most of the patients had symptoms within 2 to 4 weeks of the start of treatment (1). de Groen et al reported a patient with severe alendronate-induced esophagitis, whose clinical course, gastroscopic, and computed tomographic findings were quite similar to those of the present patient (1). On the other hand, other reported cases of esophageal stricture associated with alendronate use, presented more than 6 months after the start of treatment $(4,6)$.

On the basis of the analyses of the cases with esophageal adverse events in association with alendronate use in the literature, the postulated pathophysiologic mechanisms of esophagitis associated with alendronate use were direct esophageal irritation with prolonged local mucosal exposure to the drug, and possibly reflux of drug-containing gastric contents (1). A study using a rabbit model of alendronateinduced esophagitis showed that alendronate stimulates net ion $(\mathrm{Na})$ transport in esophageal epithelium by increasing apical membrane sodium channel activity without overt morphological changes and alteration in barrier function (8). Also alendronate is far more damaging at acidic than at neu- 
tral $\mathrm{pH}(8)$, suggesting that its association with esophagitis requires gastric acid for expression. However, direct measurement of esophageal reflux of gastric acid by esophageal $\mathrm{pH}$ monitor was not performed, we could not confirm esophageal reflux in this patient.

The sequence of events and the findings of this patient lead us to the diagnosis that he had esophagitis caused by alendronate tablet. This patient did not have risk factors for alendronate-induced esophagitis, such as inappropriate dosing, high age, preexisting reflux esophagitis, abnormalities of the esophagus that promote esophageal reflux or delay of esophageal emptying such as stricture or achalasia. Moreover, the initial symptoms suggested esophagitis occurred 48 hours after the first intake of alendronate and severe local and systemic inflammatory response, including high fever, severe chest pain, and marked elevation of C-reactive protein without esophageal perforation were observed. Therefore, we hypothesized that the esophageal event in this patient was associated not only with chemical irritation but also with delayed hypersensitivity reactions caused by alendronate.

DLST, also known as lymphocyte transformation test, is an in vitro test, which aims to detect circulating drugreactive memory $\mathrm{T}$ cells by proliferative response to an alleged drug (9-12). This test is a useful diagnostic tool to identify subjects allergic to certain drug, such as penicillins, especially in patients with delayed hypersensitivity reactions (9). The determination of the cut-off values of SI has been controversial and may differ among the drugs. Pichler and Tilch reported that an SI $>200 \%$ generally classifies the test as positive, based on negative values in exposed but not allergic individuals, though the DLST to some drugs should have a higher SI to be positive (e.g. $>300 \%$ for beta-lactam antibiotics, $>400 \%$ for vancomycin and radiocontrast materials) (10). The DLST has a general sensitivity of $60-70 \%$ and specificity of $85-100 \%$ (10). Other groups found specificities of $100 \%$ repeatedly, for example, in carbamazepine hypersensitivity and lamotrigine hypersensitivity, and of 93\% for beta-lactam hypersensitivity as exposed, while nonallergic persons showed no or only very rarely some proliferation to the drug $(11,12)$.

In the present case, in the acute phase of esophagitis, alendronate-induced mononuclear cell proliferation is extremely high $(13,714 \mathrm{cpm}$ for alendronate, $230 \mathrm{cpm}$ for negative control, and SI 5,962\%). In the remission phase (after 2.5 months), the proliferative response to alendronate was reduced (623 cpm, SI $223 \%$ ), though that to negative control was almost unchanged $(279 \mathrm{cpm})$. This case report provides the first evidence of the presence of alendronatereactive $\mathrm{T}$ cells in the peripheral blood of patients with alendronate-induced esophagitis and insight into the role of hypersensitivity reactions to alendronate in the pathogenesis of alendronate-induced esophagitis. Further studies are needed to confirm the involvement of $\mathrm{T}$ cell-mediated delayed hypersensitivity reaction to alendronate in the pathogenesis of esophagitis caused by alendronate.

\section{References}

1. de Groen PC, Lubbe DF, Hirsch LJ, et al. Esophagitis associated with the use of alendronate. N Engl J Med 335: 1016-1021, 1996.

2. Castell DO. "Pill esophagitis"--the case of alendronate. N Engl J Med 335: 1058-1059, 1996.

3. Lieberman UA, Hirsch U. Esophagitis and alendronate. N Engl J Med 335: 1069-1070, 1996.

4. Naylor G, Davies MH. Oesophageal stricture associated with alendronic acid. Lancet 348: 1030-1031, 1996.

5. Colina RE, Smith M, Kikendall JW, Wong RK. A new probable increasing cause of esophageal ulceration: alendronate. Am J Gastroenterol 92: 704-706, 1997.

6. Ryan JM, Kelsey P, Ryan BM, Mueller PR. Alendronate-induced esophagitis: case report of a recently recognized form of severe esophagitis with esophageal stricture--radiographic features. Radiology 206: 389-391, 1998.

7. Bauer DC, Black D, Ensrud K, et al. Upper gastrointestinal tract safety profile of alendronate: the fracture intervention trial. Arch Intern Med 160: 517-525, 2000.
8. Dobrucali A, Tobey NA, Awayda MS, et al. Physiological and morphological effects of alendronate on rabbit esophageal epithelium. Am J Physiol Gastrointest Liver Physiol 283: G576-G586, 2002.

9. Luque I, Leyva L, Jose Torres M, et al. In vitro T-cell responses to beta-lactam drugs in immediate and nonimmediate allergic reactions. Allergy 56: 611-618, 2001.

10. Pichler WJ, Tilch J. The lymphocyte transformation test in the diagnosis of drug hypersensitivity. Allergy 59: 809-820, 2004.

11. Naisbitt DJ, Farrell J, Wong G, et al. Characterization of drugspecific $\mathrm{T}$ cells in lamotrigine hypersensitivity. J Allergy Clin Immunol 111: 1393-1403, 2003.

12. Naisbitt DJ, Britschgi $M$, Wong G, et al. Hypersensitivity reactions to carbamazepine: characterization of the specificity, phenotype, and cytokine profile of drug-specific T-cell clones. Mol Pharmacol 63: 732-741, 2003.

(C) 2008 The Japanese Society of Internal Medicine

http://www.naika.or.jp/imindex.html 